\title{
The Quantization of Black Holes, Lower Mass Limit, Temperature, and Lifetime of Black Holes in a Simple Way
}

\author{
Sirus Arya Enejad \\ Physics Department, Eastern Illinois University, Charleston, Illinois, USA \\ Email: golayle1111@gmail.com
}

How to cite this paper: Enejad, S.A. (2021) The Quantization of Black Holes, Lower Mass Limit, Temperature, and Lifetime of Black Holes in a Simple Way. International Journal of Astronomy and Astrophysics, 11, 1-10.

https://doi.org/10.4236/ijaa.2021.111001

Received: September 8, 2020

Accepted: January 8, 2021

Published: January 11, 2021

Copyright () 2021 by author(s) and Scientific Research Publishing Inc. This work is licensed under the Creative Commons Attribution International License (CC BY 4.0).

http://creativecommons.org/licenses/by/4.0/

\begin{abstract}
We have shown that the permittivity of space grows for a beam of light as the gravitational field increases. Also, we have derived two values for Chandrasekhar limit. Using the necessity of equality of wavelengths in matching systems, we have derived the Hawking black hole temperature and evaporation time in an easier and completely different way, and shown that mass and wavelength of the field and black hole at Schwarzschild sphere are quantized. The extreme simplicity of the present new approach to black holes compared to those based on general relativistic ones should promote it.
\end{abstract}

\section{Keywords}

Black Holes

\section{Introduction}

To basically find the lifetime of a black hole, S. Hawking has suggested (in 1974) that space is formed of particles (or antiparticles) of positive energy and virtual antiparticles (or particles) of equal, in absolute value, of negative energy, as Kip Thorne mentions in his book [1] [2]. Hawking said that the immediate space to the event horizon constantly decomposes to its real and virtual particle - antiparticle. The virtual antiparticles (with negative energy) fall into the black hole and the real particles escape to infinitely far from it.

According to his suggestion, those negative energy virtual antiparticles falling into the black hole immediately get together with the positive energy real particles underneath the event horizon and reconstruct space. In this manner, the radius of the black hole event horizon should be decreasing. Considering the Hawking black hole evaporation time, this reduction is extremely slow. Thus, 
this model derives a time as of evaporation (or, lifetime) of a black hole. However, we contend that the assumption of space include negative energy antiparticles should merely be an imaginary one. Also, the gravitational field is not addressed in this hypothesis, because as we know the space around a black hole is filled with strong gravitational energy.

Here, we first look at the motion of a photon of light in a gravitational fields especially, that of a black hole. Next, we will aim to remove the problem and even not talk about negative energy particles. Also, we show agreement between the present results and Chandrasekhar limit plus Hawking - black hole temperature and evaporation time.

\section{The Principle of Harmony and Light and Matter in the Vicinity of a Black Hole}

First, we postulate that two waves get to "match" and become united once they have the same wavelength. We call this the principle "harmony." [3] (Appendix 1)

We will show, for black holes next to their event horizon sphere the energy (quantum) within it is made to match its gravitational field there. This matching results in the gravitational field to absorb the energy units and make the neutral space. Eventually the energy of the gravitational field will become equal to zero also. There will no longer remain any energy inside the shrinking black hole. This is our suggested way of evaporation of a black hole. The time length measured by us (on the surface of the earth) that the gravitational field takes to absorb $M c^{2}$ from the inside of the event horizon sphere is actually the black hole lifetime, which we call " $\tau_{\text {total }}$ " The advantage of this model is contrary to the assumption of negative - energy particle or antiparticle; the gravitational field is an established experimental reality.

First, however we determine the ratio of energy, $W_{p h}$, (i.e. the energy of the photon from the standpoint of an observer in free space and not the curved space), while the photon is moving radially toward the sphere of radius $r_{s}$ $\left(2 G M / c^{2}\right)$ of a black hole, to its (the photon's) vacuum energy, $W_{0}$. Here, $G(6.67$ $\times 10^{-8} \mathrm{dyn} \cdot \mathrm{cm}^{2} / \mathrm{g}^{2}$ ) is the universal gravitational constant. The gravitational potential energy, $W_{p h}$, of a photon of light (of vacuum energy $W_{0}$ ) is:

$$
W_{p h}=\frac{W_{0}}{c^{2}} v^{2}=\frac{G M}{r} \frac{W_{0}}{c^{2}}
$$

According to virial theorem this $\left(-G M W_{0} / c^{2} r\right)$ is half of the energy that the photon loses. The other half is already radiated to space at infinity or inside of the black hole.

Although Stefano and Quattrini [4], on the basis of a not enough exact experiment by NASA, for our case, deny the existence of a gravitational potential. But more recently Alexandre Chaloum Elbeze [5] clearly talks about the effect of gravitational field on the motion of photons.

Consequently, at any $r$, the total energy of the photon is:

$$
W_{p h}=W_{0}-\frac{2 G M W_{0}}{c^{2} r} .
$$


The permittivity of space is:

$$
\varepsilon=\frac{W_{0}}{W_{p h}} .
$$

Therefore,

$$
\begin{gathered}
\varepsilon=\frac{W_{0}}{W_{0}-2 G M W_{0} / c^{2} r} \Rightarrow \varepsilon=\left(1-\frac{2 G M}{c^{2} r}\right)^{-1} \\
\Rightarrow n=\left(1-\frac{2 G M}{c^{2} r}\right)^{-1 / 2} .
\end{gathered}
$$

This increase in index of refraction is the reason for slowing down and bending a photon in gravitational field. Then, we can calculate the speed, frequency, and wavelength of any incoming light. We have the following

$$
\begin{gathered}
n=\frac{c}{v} \Rightarrow v=c\left(1-\frac{2 G M}{c^{2} r}\right)^{1 / 2} . \\
\frac{f_{0}}{f}=\frac{h f_{0}}{h f}=\frac{W_{0}}{W_{p h}}=\varepsilon \Rightarrow f=f_{0}\left(1-\frac{2 G M}{c^{2} r}\right) .
\end{gathered}
$$

And thus:

$$
\lambda=\lambda_{0}\left(1-\frac{2 G M}{c^{2} r}\right)^{-1 / 2}
$$

As $r \rightarrow r_{s}$ the speed $v$, frequency $f$, and wavelength $\lambda$, of an incoming photon, respectively go to zero, zero, and infinity. Consequently, at $r_{s}$, any incoming photon of light would have zero frequency (infinite wavelength). According to Equation (2), the total energy, $W_{p h}$ left for any incoming photon of light will go to zero, as $r \rightarrow r_{s}$ or:

$$
W_{p h}\left(r_{s}\right)=0 .
$$

So, a photon of light stops existing at the Schwarzschild radius of a black hole having zero speed and having given up all its free-space energy $W_{0}$. Time stops as $r \rightarrow r_{s}$ thus, the period of any photon of light goes to infinity. Therefore, no motion is conceived inside the sphere of radius $r_{s}$ and the contraction of the black hole should stop inside this sphere.

The situation is different for an incoming mass at $r_{s}$. Putting the kinetic energy of the mass equal to the gravitational potential we find the speed to be equal to that of light $(c)$ at $r_{s}$. By the time the particles of matter reach the Schwarzschild sphere they have radiated almost quarter of their mass (matched to the gravitational field) and according to virial theorem the other quarter is radiated most probably inside the system of the particle - black hole. Thus, the three quarters of the mass join the black hole.

\section{The Derivation of Minimum Mass of a Protosun and a Neutron Star. Comparison with Chanrasekhar Limit}

At this point let us derive the minimum possible value of the mass of the core of 
a star that can change to a neutron star and eventually to a black hole.

The first way we suggest to find this value is; we claim that, in order for a star to be able to evolve to a neutron star is; at the beginning of the star's formation all the kinetic energy of the electron of every Hydrogen $(\mathrm{H})$ atom at its ground state, i.e. $13.6 \mathrm{eV}$ plus that already radiated (which itself is $13.6 \mathrm{eV}$ ) have to be equal to the star's self - gravitational field of the core at the surface of the proto star. Here we include the radiated part of the electron because this energy is most probably saved in the other parts of the proto - star itself. In essence we say, our requirement for a star to eventually evolve to a neutron star is that all of the electrons collapse in its protons of the core and all of the core's $H$ atoms ground state orbital electrons' kinetic energies plus those already radiated, to be equal to the star's self-gravitational field (as a whole).

According to a paper by D.Ezer and A.G.W. Cameron [6] "The maximum radius of the protosun consistent with gravitational stability is $57 R_{\odot}$.”

The self - gravitational energy of a spherical star of mass $M$ is

$$
W_{S E L F}=\frac{3 G M^{2}}{5 r_{i}} \text {. }
$$

Then, requiring the self - gravitational energy of core but at the surface of the protosun to be equal to $2 \times 13.6 \mathrm{eV}$ times $N\left(M_{\text {core }} / m_{\text {Hydrogen }}\right)$ then we claim $M_{\text {core }}$ would be big enough to form a neutron star [7]. Because first it is the core of a star that survives the supernova explosion and converts to a white dwarf and second in the beginning the core is made of $\mathrm{H}$ atoms.

In that case, many ground state electrons would not have any orbital kinetic energy left and thus, ideally collapse into their protons. Therefore, there would be all neutrons in the environment. We have:

$$
2 \times 13.6\left(1.6 \times 10^{-12}\right) \frac{M_{\text {core }}}{m_{\text {Hydrogen }}}=W_{\text {Self-Gravitation }}(\text { of the core }) .
$$

where, $W_{\text {Self-Gravitational }}$ is in ergs and $m_{\text {Hydrogen }}\left(1.67 \times 10^{-24} \mathrm{~g}\right)$. Assuming $r_{\text {protosun }}=$ $60 R_{\odot}\left(\approx 4.18 \times 10^{12} \mathrm{~cm}\right)$ instead of $57 R_{\odot}$, from the above two equations we have:

$$
\begin{gathered}
2 \times 13.6\left(1.6 \times 10^{-12}\right) \frac{M_{\text {core }}}{1.67 \times 10^{-24}}=\frac{3 G M_{\text {core }}^{2}}{5 r_{\text {protosun }}} \\
\Rightarrow M_{\text {core }}=2.72 \times 10^{33} \mathrm{~g}=1.37 M_{\odot} .
\end{gathered}
$$

where $M_{\odot}\left(1.99 \times 10^{33} \mathrm{~g}\right)$ is the mass of the sun. Therefore, the minimum mass limit for the core of a star to eventually turn to a completely stationary electron proton system is $M_{\text {core }}=1.37 M_{\odot}$.

Second, to show an alternative way of finding the lower limit mass of a neutron star we need to declare that:

The fine structure constant $\alpha\left(e^{2} / \hbar c=136.7^{-1}\right)$ is equal to $\left(m_{\text {electron }} / \pi^{2} m_{\text {proton }}\right)^{1 / 2}$ which is $0.51 \%$ different than $\alpha$.

Under the extremely high pressure and temperature of the core it is possible that some neutrons turn into antineutrons. Therefore, the interaction between a 
neutron and antineutron to take place or, in case of the neutron decay to hyperons two neutrons are treated as two interactions of $+e$ and $-e$ like in the $\mathrm{H}$ atom. But particles here would have the same mass. Then, the factor,

$$
m_{\text {electron }} / \pi^{2} m_{\text {proton }} \text { converts to } m_{\text {neutron }} / \pi^{2} m_{\text {neutron }}=1 / \pi^{2} \text {. }
$$

The lower limiting mass of a neutron star that converts to a black hole is found by assuming that every neutron has paired with an antineutron or, hyperons to join each other. So the kinetic energy (quantum mechanically) amounts to $2\left(m_{n} c^{2} / \pi^{2}\right)$. This energy, times the number of the pairs in the neutron star is the self - gravitational energy of the star:

$$
\begin{aligned}
2 \times \frac{m_{n} c^{2}}{\pi^{2}} \frac{M_{\text {neutron star }}}{2 m_{n}} & =\frac{3 G M_{\text {neutron star }}^{2}}{5 r_{\text {neutron star }}} \Rightarrow \frac{M_{\text {neutron star }}}{r_{\text {neutron star }}}=2.28 \times 10^{27} \\
& \Rightarrow M_{\text {neutron star }}=1.37 M_{\odot} .
\end{aligned}
$$

The radius of a typical neutron star is reported as between 8 to $12 \mathrm{~km}$ [8]. Taking the $r_{\text {neutron star }}$ as $12 \mathrm{~km}$ then the lower limit value of the mass of a neutron star is $M_{\text {neutron star }}=1.37 M_{\odot}$. If the mass of the core is bigger than a natural number times $1.37 M_{\odot}$ then the extra mass will be expelled to space during the supernova explosion of the star to make the mass of the neutron star an exact natural number times $1.37 M_{\odot}$. Thus, we think the mass of the black holes is Quantized and is $M=n \cdot 1.37 M_{\odot}$.

Following Equations ((11), (12)) the average of the two limiting masses, is: $M_{\text {neutrn star }} \equiv M_{0}=1.37 M_{\odot}$.

The Chandrasekhar minimum mass limit for a white dwarf that can turn to a neutron star is $M=1.4 M_{\odot}$, [9] [10] which is less than $2.2 \%$ bigger than our value. When we find the minimum mass of a neutron star that turns to a black hole in fact, we have found the minimum mass limit for a white dwarf that is able to turn to a neutron star.

Because "time" is frozen at the event horizon of a black hole there should not be any further mass implosion. Thus, all the pairs of neutrons - antineutrons, or hyperons, should remain stationary or change to energy underneath the event horizon sphere (especially) under the extremely high pressure and temperature of the neutron star). Consequently, at this point there is no further implosion, and we believe no mass is left but there is energy. This energy is equal to $n M_{0} c^{2}$, i.e. that of the black hole. Thus, because time is frozen after the event horizon sphere there is not any matter inside a black hole but energy. This energy is stationary and stays inside this sphere. This is our structural model for all gravitational black holes of universe.

\section{Wavelength of the Gravitational Field and That of Black Hole Next to Shwarzschild Sphere. The Derivation of Temperature and Lifetime of a Black Hole. Comparison with Hawking Results}

Now let's calculate the temperature as well as the "lifetime" of black holes. We 
claim that the gravitational field and the inside of the black hole next to sphere of radius $r_{s}$ match and make the neutral space.

To get the wavelength of the gravitational field as well as that of the stationary energy inside the event horizon sphere we proceed as follows. Looking at the gravitational field immediate to the event horizon, it should be spherical and should be a wave wrapped around the closed spheres of the event horizon. The mass of the black hole in general being $n M_{0}$ and referring to quantum mechanical de Broglie's principle, we claim at exactly $4 \pi r_{s} / n\left(=\lambda_{0}\right)$ as many as $n$ of $\lambda_{0}$ should exist. We put $4 \pi$ (instead of $2 \pi$ ) here because the gravitational waves are spherical.

Requiring that the wavelength of energy inside the event horizon sphere be the same. The wavelength is: $\lambda_{0}$ of energy inside event horizon $=4 \pi r_{s} / n$ too. Therefore, the field immediately outside event horizon will have the same wavelength as the inside stationary trapped energy and according to principle of harmony match it.

For energy waves inside the Schwarzschild sphere we take $E=h f$ and $\lambda_{G-e} f_{G-e}=c$. Here the $h\left(6.62 \times 10^{-27} \mathrm{~cm}^{2} \cdot \mathrm{gm} / \mathrm{s}\right)$ is Plank's constant and the subscript " $G-e$ " indicates "the Gravitational field outside and the energy inside and immediate to the event horizon sphere. In that case $f_{G-e}=n c / 4 \pi r_{0 s}$ and $E=\hbar c^{3} / 4 G M$. Note that, the energy inside is frozen (in time)and there is no motion thus, no real frequency exists for this energy wave, however we accept the equation $\lambda_{G-e} f_{G-e}=c$ and we argue that if there was pure energy in empty space then this equation would be valid. Later, we will show more evidence to the validity of this assumption. Thus, the energy is $w_{Q} \equiv \hbar c / 2 r_{s}$ or:

$$
w_{Q}=\frac{\hbar c^{3}}{4 G M}=w_{0} .
$$

Here $w_{Q}$ is the energy of the black hole on the inner surface of the Schwarzschild sphere. Note that $w_{Q}=w_{0}$.

We take the quantum of energy equal to $\left(w_{0} / 4 \pi\right)=\left(\hbar c^{3} / 16 \pi G M\right)$ i.e. the energy per squares of area $r_{0 s}^{2}$ on the spheres of radius $r_{s}$. Where $M\left(=n M_{0}\right)$ is the black hole mass. We put this unit energy equal to $(1 / 2) k_{b} T$. With $k_{b}(1.38 \times$ $\left.10^{-16} \mathrm{erg} \cdot \mathrm{K}^{-1}\right)$ the Boltzmann constant and $T$ the absolute temperature of the black hole. The reason for the one-dimensional factor of $1 / 2$ (and not three dimensional factor of 3/2) for $k_{b} T$ is that the exchange of heat between the black hole and outside takes place on one dimensional circles (for some black holes, of accretion disk).

Taking the quantum of energy $\left(w_{0} / 4 \pi\right)$ equal to $(1 / 2) k_{b} T$ we have:

$$
T_{\text {our value }}=\frac{\hbar c^{3}}{8 \pi G M k_{b}} \text {. }
$$

On the other hand, the Hawking radiation temperature, $T_{\text {Hawking }}$ is:

$$
T_{\text {Hawking }}=\frac{\hbar c^{3}}{8 \pi G M k_{b}} .
$$


The $T_{\text {our value }}$ is the same as $T_{\text {Hawking }}$ or, there is a $100 \%$ agreement between the two values. Therefore, the assumption of the inside the event horizon wavelength being $\lambda$ of energy inside event horizon $=4 \pi r_{s}$ or $\lambda_{G-e}=4 \pi r_{s}$ seems reasonable.

For $M_{0}=1.4 M_{\odot}=1.4 \times 1.99 \times 10^{33} \mathrm{~g}=2.79 \times 10^{33} \mathrm{~g}$, i.e. the Chandrasekhar limit:

$$
\lambda_{0}=4 \pi r_{0 s}=\frac{8 \pi G}{c^{2}} M_{0} \approx 5.20 \times 10^{6} \mathrm{~cm} .
$$

And $T_{\text {our value }}=T_{\text {Hawking }}$ is:

$$
T_{\text {our value }}=T_{\text {Hawking }}=\frac{\hbar c^{3}}{8 \pi G M_{0} k_{b}}=4.39 \times 10^{-8} \mathrm{~K} .
$$

And for all black holes immediately bellow the event horizon the quantum of energy is:

$$
w_{0 Q}=\frac{\hbar c^{3}}{4 G M_{0}} \approx 3.81 \times 10^{-23} \mathrm{ergs} .
$$

With $M\left(M_{0}=1.4 M_{\odot}\right.$, the least mass of the black hole) in grams.

Immediately after its birth, the aging of a black hole starts. One unit of energy $w_{0 Q}$ from the inside of event horizon sphere matches the gravitational field making the space. Taking the self-gravitational energy of the black hole as that classical value of it $W_{G}=-3 G M^{2} / 5 R$ then, at the event horizon $r_{s}^{\prime}=2 G\left(M-\left|w_{0 Q}\right| / c^{2}\right) / c^{2}$ note that the negative sign for $W_{G}$ is indicative of its attractive nature and does not mean that this energy is negative. We have

$$
\begin{gathered}
W\left(\text { at } r_{s}^{\prime}\right) \approx-\frac{3}{5} \frac{G\left(M-w_{0 Q} / c^{2}\right)^{2}}{r_{s}^{\prime}}=\frac{3}{5} \frac{-G\left(M-w_{0 Q} / c^{2}\right)^{2}}{2 G\left(M-\left|w_{Q}\right| / c^{2}\right) / c^{2}} \\
\Rightarrow W\left(\text { at } r_{s}^{\prime}\right) \approx-\frac{3}{10} M c^{2}+\frac{3}{5} w_{0 Q} .
\end{gathered}
$$

According to virial theorem one half of $\left(w_{0 Q}\right) / 2$ gets to be radiated (perhaps back to the black hole). Thus, the energy of the field is increased by $3\left(w_{0 Q}\right) / 10$. In Equation (19) we have ignored the term $\left(w_{0 Q}\right)^{2}$ versus $M^{2}$ in the numerator and ignored the term $\left|w_{0 Q}\right|$ versus $M$ in the denominator. Therefore, once we have an energy at the event horizon radius then only three $10^{\text {th }}$ of it has to be already invested in the gravitational field while the other seven $10^{\text {th }}$ is with the black hole, or that energy itself is a part of the black hole, but is debilitated to match with the gravitational field. As result, the total energy of the field increases by $3 w_{0 Q} / 10$.

Next, the gravitational field absorbs another unit of energy trapped inside the black hole. Now, the field's energy increases by another $3\left(w_{0 Q}\right) / 10$. This flow of $w_{0 Q}$ from inside the event horizon to the gravitational field, continues until all of the trapped energy inside the black hole (i.e. its $M c^{2}$ ) flows to the gravitational field. The $7\left(w_{0 Q}\right) / 10$ 's of the $w_{0 Q}$ 's make their own gravitational field after the 
original gravitational field is exhausted and so on. Eventually, the gravitational fields energies match the entire $M c^{2}$ of the black hole. At this stage, the energy of the gravitational fields and that trapped inside the black hole will both become equal to zero, meaning the black hole will be completely evaporated and its gravitational field will be completely gone too.

Let us find now the total time $\left(\tau_{\text {total }}\right)$ taken for the gravitational fields to match a total energy of the black hole.

The unit energy of the surface immediately underneath the Schwarzschild surface is $w_{0 O}=\hbar c^{3} / 16 \pi G M$ ergs. The "period" we find to this energy is $T_{G-f}=32 \pi^{2} G M / c^{3}$. Thus, the time rate of reduction in mass is $(3 / 10)$ energy unit / the time period we ascribe to its radiation.

The following relation holds:

$$
c^{2} \frac{\mathrm{d} \mu}{\mathrm{d} t}=-\frac{3}{10} \frac{\hbar c^{3} / 16 \pi G \mu}{32 \pi^{2} G \mu / c^{3}}=-\frac{3}{10} \frac{\hbar c^{6}}{512 \pi^{3} G^{2} \mu^{2}} .
$$

where the factor $3 / 10$ in above eq. indicates energy matches as much as the gravitational and $\mu$ is the reducing mass. Thus:

$$
\mu^{2} \mathrm{~d} \mu=-\frac{3}{10} \frac{\hbar c^{4}}{512 \pi^{3} G^{2}} \mathrm{~d} t .
$$

Integrating the left side from $M$ to 0 and the right side from 0 to $\tau_{\text {total }}$ (i.e. the lifetime of black hole) we have:

$$
\tau_{\text {total }}=\frac{\pi^{2}}{9} \frac{5120 \pi G^{2}}{\hbar c^{4}} M^{3} .
$$

where, again $M$ is in grams and $\tau_{\text {total }}$ in seconds is the total time it takes to pull a trapped energy $M c^{2}$ out of the black hole i.e. $\tau_{\text {total }}$ is our calculated total lifetime of black hole.

The Hawking "total lifetime" of a black hole is

$$
T_{\text {Hawking }}=\frac{5120 \pi G^{2}}{\hbar c^{4}} M^{3} .
$$

Comparing the right hand sides of (22) and (23) both of these times, are proportional to $M^{\hat{p}}$. We have $T_{\text {Hawking }} / \tau_{\text {total }} \approx 1.3 \%$.

\section{Discussion}

Albert Einstein has said "everything has to be made as simple as possible but not simpler". In this paper we present a simple method of deriving black hole constants which agree with previous ones, a method that does not need Einstein equations [11] [12]. In this process we only use the principle that when two waves have the same wavelength can unite [3] (Appendix 1).

We have introduced quantized wavelengths of the gravitational field and the black hole at the Schwarzschild sphere, that there is energy and not mass inside a black hole, and quantized the energy of black holes. In future papers we talk about the quantization of black holes in more detail. 


\section{Conclusion}

In a completely different and much easier way, we have derived the Chandrasekhar mass limit within about $10 \%$, the Hawking temperature of a black hole exactly, the Hawking black hole lifetime within less than $1.5 \%$, presented the quantized wavelengths of the gravitational field and the black hole next to the Schwarzschild sphere, and have quantized the energy of a black hole.

\section{Acknowledgements}

The author would like to thank professors Sadegh and Nader Angha for their immense contributions, especially on this paper.

\section{Conflicts of Interest}

The author declares no conflicts of interest regarding the publication of this paper.

\section{References}

[1] Hawking, S.W. (1988) A Brief History of Time. Bantam, New York. https://doi.org/10.1063/1.2811637

[2] Thorne Kip, S. (1994) Black Holes \& Time Wraps. W. W. Norton, New York.

[3] Angha, N. (2000) Expansion \& Contraction within Being. M.T.O Shahmaghsoudi, Riverside, Ca.

[4] Stefano, Q. (2014) The Gravitational Potential Energy of Photons Is Against the Experimental Evidence. 3 September.

[5] Elbeze, A.C. (2018) On Gravitational Waves: Did We Simply Detect the Gravitational Effect of the Sun on the Photons Moving in the Cavity of Interferometers LIGO and VIRGO. Journal of Modern Physics, 9, No. 6.

[6] Ezer, D. and Cameron, A.G.W. (1963) The Early Evolution of the Sun. Icarus, 1, 422-441. https://doi.org/10.1016/0019-1035(62)90045-3

[7] Shipman, H.L. (1979) Masses and Radii of White-Dwarf Stars. III - Results for 110 Hydrogen-Rich and 28 Helium - Rich Stars. The Astrophysical Journal, 228, 240-256. https://doi.org/10.1086/156841

[8] Haensel, P., Potekhin, A.Y. and Yakovlev, D.G. (2007) Neutron Stars. Springer, New York.

[9] Chandrasekhar, S. (1931) The Maximum Mass of Ideal White Dwarfs. Astrophysical Journal, 74, 81. https://doi.org/10.1086/143324

[10] Chandrasekhar, S. (1935) The Highly Collapsed Configurations of a Stellar Mass (Second Paper). Monthly Notice of the Royal Astronomical Society, 95, 207. https://doi.org/10.1093/mnras/95.3.207

[11] Ohanian, H.C. (1976) Gravitation and Spacetime. W. W. Norton, NY.

[12] Adler, R.J., Bazin, M.J. and Schiffer, M. (1965) Introduction to General Relativity. 2nd Edition, McGraw Hill, New York. 


\section{Appendix 1}

The principle harmony introduced by Prof. N. Angha says that two systems that have the same wavelengths can match and make a wave with twice as big intensity i.e. two of the SAME frequency and thus, wavelength. 